Relations industrielles

Industrial Relations

\title{
Trade-Union Membership, 1897-1962, par Leo Troy. Occasional Paper 92. National Bureau of Economic Research, New York, 1965. 22 pages et 65 pages de tableaux.
}

\section{Gérard Dion}

Volume 21, numéro 3, 1966

URI : https://id.erudit.org/iderudit/027724ar

DOI : https://doi.org/10.7202/027724ar

Aller au sommaire du numéro

Éditeur(s)

Département des relations industrielles de l'Université Laval

ISSN

0034-379X (imprimé)

1703-8138 (numérique)

Découvrir la revue

Citer ce compte rendu

Dion, G. (1966). Compte rendu de [Trade-Union Membership, 1897-1962, par Leo Troy. Occasional Paper 92. National Bureau of Economic Research, New York, 1965. 22 pages et 65 pages de tableaux.] Relations industrielles / Industrial Relations, 21(3), 471-471. https://doi.org/10.7202/027724ar

Tous droits réservés @ Département des relations industrielles de l'Université Laval, 1966
Ce document est protégé par la loi sur le droit d'auteur. L'utilisation des services d’Érudit (y compris la reproduction) est assujettie à sa politique d'utilisation que vous pouvez consulter en ligne.

https://apropos.erudit.org/fr/usagers/politique-dutilisation/ 
enrichir la bibliothèque de toute personne intéressśe à la gestion de l'entreprise. II constitue une sorte de vade mecum sur le sujet. Et ceci serait encore plus vrai, si l'auteur avait référé le lecteur aux ouvrages les plus importants sur les principaux aspects traités. Car le livre de M. Pugsley ne contient pas de bibliographie et les renvois aux ouvroges de base sont très rores.

\section{Bertrand BELZILE}

Trade-Union Membership, 1897-1962, par Leo Troy. Occasional Paper 92. National Bureau of Economic Research, New York, 1965. 22 pages et 65 pages de tableaux.

Cet ouvrage reprend un article original paru sous le même titre dans The Review of Economics and Statistics (February1965) en y ajoutant, cependant, en appendice 65 paces de tobleaux.
Une première partie décrit les grandes tendances dans les effectifs syndicaux aux Etats-Unis; la seconde est consacrée aux sources et méthodes de calcul; la troisième compare et discute la méthode de compilation utilisée avec celle du Bureau of Labor Statistics; enfin, dans la quatrième, l'auteur présente une série de suggestions pour une amélioration des statistiques des effectifs syndicaux.

Nul ne conteste l'importance des statistiques syndicales. Qui d'ailleurs, dans l'élaboration d'un travail dans le domaine des relations du travail ne s'est pas trouvé handicapé par l'absence de données adéquates? Pour les obtenir, il faudrait plus de collaboration entre divers organismes gouvernementaux et oussi de la part des groupements syndicaux eux-mêmes. Cela est vrai non seulement aux Etats-Unis, mais aussi au Canada.

Gérard DION

\section{SOCIALISATION ET RELATIONS INDUSTRIELLES}

La socialisation: caractere et signtfication (GERARD DION). Lentreprise priote face d la socialisation (RAYMOND GERIN). Le syndicalisme on contexte socialisée: fonctions ot responsabilites nouvelles (LOUIS-MARIE TREMBLAY). Le role de lEtat en relations du travail - essai de rb́foaluation (JEAN-REAL CARDIN). L'Etat-employeur et la fonction publique (S.J. FRANKEL). Discussion (ALBERT GINGRAS, YVON CHARTRAND, MICHEL HARRISON). La négociation collective dans les secteurs priós rubventionnés par lEtat. Point de oue patronal (PAUL DESROCHERS). Point de oue syndical (JACQUES ARCHAMBAULT). Discussion (GILLES GAUDREAULT, LEOPOLD GARANT, J.-R. GAUTHIER). Planification, entreprise privée et syndicalisme libre (PAUL NORMANDEAU).

1 volume, 188 pages - Prix: $\$ 3.00$

LES PRESSES DE L'UNIVERSITE LAVAL 